\title{
Developing Collaborative Social Software
}

\author{
Ismail Bhana and David Johnson \\ Advanced Computing and Emerging Technologies Centre, \\ School of Systems Engineering, \\ The University of Reading, \\ Reading, RG6 6AY, United Kingdom \\ \{i.m.bhana, d.johnson\}@reading.ac.uk
}

\begin{abstract}
The Internet has an increasing role in facilitating communication between people and groups of people. As access to the Internet and World Wide Web is widely available, collaborative services enabled over the Internet are also burgeoning. In this paper, we present the current issues and our techniques for developing collaborative social software. We discuss online communities in the context of social collaborative systems. We then describe our approach to the development of supporting software for online communities and collaboration.
\end{abstract}

\section{Introduction}

The Internet has an increasing role in facilitating communication between people and groups of people. Traditional forms, such as telephones, mail and even face-to-face meetings, are gradually being assumed by computer based forms where socialisation is enhanced through rich media sharing, reflection on past experiences and greater availability of services. Communication software allows for audio conferencing, video conferencing, as well as real-time application sharing. Reflection is supported by allowing the extensive logging of communications and the ability to annotate these logs. Content sharing can provide instant publishing of and access to information. As access to the Internet and World Wide Web (WWW) is widely available, collaborative services enabled over the Internet are also burgeoning.

In this paper, we present the issues and techniques for developing collaborative social software. In section 3 we discuss online communities and our approaches to supporting such communities. We then go on to describe our approach to the development of supporting software for online interaction and collaboration.

\section{Related Work}

$\mathrm{ABC}$ [1] is an exploration into how to conceptualise and model human activities in the context multi-user interactions. ABC consists of a Java-based cross-platform development framework and supporting runtime infrastructure on which applications supporting both synchronous (i.e. real-time) and asynchronous cooperation or 
collaboration can be built. $\mathrm{ABC}$ focuses on activity sharing rather than the application sharing that is more commonly found in CSCW.

Friendster [2] is a Web-based Internet social networking service that supports online communities with shared content (pictures, videos, and Blogs) and online profiles that are accessible to other community members. The objective is to enable individuals to connect, form social networks and interact in virtual communities that are linked by shared acquaintances. Friendster is based on the circle of friends technique for networking individuals in virtual communities and has its roots in Ryze [3], a business networking site founded in 2001.

The DreamTeam framework [4] consists of an environment that is designed to facilitate the rapid development of collaborative distance learning applications. The motivation behind DreamTeam is to hide the complexity of the underlying network and to enable developers to build collaborative applications transparently, using the facilities and services of the DreamTeam runtime infrastructure.

\section{Online Community}

Several common features characterise an online community or 'networked communities' [6]:

- Technological mediation of communications.

- Persistence of the overall context or activity of the community.

- Multiple interaction styles allowing entities to communicate in a range of ways.

- Real-time interaction capabilities facilitating spontaneous collaborations.

- Supporting multiple users as opposed to one-to-one contacts.

We identify three interaction styles for communication that encompass all of the aforementioned factors:

- One-to-one - Direct communication from one participant to another. Examples of this include email, audio/video chat and instant messaging (IM).

- One-to-many - A single participant communicating with multiple participants. Most commonly seen examples of this are publishing information on the WWW in the form of personal web-pages or Blogs. Other examples include audio/video conferencing.

- Many-to-many - Multiple entities communicating with each other in a larger context, for example collaborative authoring in Wikis [7] and knowledge sharing in discussion forums.

In building online communities, both synchronous and asynchronous communication can play a role. Ad hoc collaborations enable entities to self organise and communicate in real-time, as is the norm in audio/video/IM chat and conferencing. The ability to edit or annotate content allows entities to communicate over greater lengths of time where organisation of real-time meetings cannot be realised, or the nature of the task requires drawn-out periods to complete. Examples of persistence include publishing information on the WWW using Wikis, discussion forums and Blogs. 
Issues of identity also come into play as community building, whether online or not, require contributions from and collaboration between identifiable individuals. Establishing an identity is pivotal in building relationships with others within an online community, especially if there is no pre-existing relationship outside the community context. For example, an online community may bring together entities with no experience of each other but on the other extreme, a community might be formed that mirrors existing relationships in the 'real' world. In the latter case, establishing identity is not always necessary.

We identify three enablers for supporting identity in an online community:

- Profiles - A profile represents a persona that a participant wishes to project to the community.

- Portfolios - A record of participant's experiences. A portfolio represents achievements, experience, and evidence, including reflections on each of these. Dependant on the context, parts of portfolios are publishable to the community for others to form opinions.

- Participations - The process of interacting with other community entities. By communicating and collaborating with others, one builds an identity purely through interaction, rather than publishing some record of identity. As community participants form opinions of each other, social norms, reputations and individual's roles can be defined. Norms and roles are not static once identified, and can change as collective experiences go on.

As an individual builds up a persona defined by their experiences through contributing, communicating and collaborating with others in a community, issues regarding intellectual property arise. Typically information is centralised. Users' profiles, portfolios and content tend to reside in one place so that access may be tightly controlled; this is normally the case with Web-based community services. However, there is a growing need to be able to share and exchange information to support community interactions, such as personal data and content, between multiple different community systems (Websites or applications) transparently and provide services that unify various user profiles, portfolios and participations across multiple systems.

In most cases, access to profile, portfolio and participation data is controlled by the service provider for a particular community system, forum or community website (with limited user control). Most service providers require users to register and agree to certain terms and conditions of service that specify how user information is handled by that provider alone. This has the affect of hindering the process of interoperation of community service where there is no dialog between providers (or where services are hosted by competing organisations). Options here do exist, such as Microsoft Passport [8], but there is a need for open specifications and technology to facilitate the transference of user data (as we have defined above) transparently between services and community systems - ideally where it is managed and controlled by the participants themselves.

Depending on the needs of a service provider, we submit that a balance between the needs of individual community systems to enable meaningful contextual 
interactions and freedom of the participants to control access to profile, portfolio and participation data transparently across multiple community systems is needed.

\section{Collaborative Communities with Coco}

Coco [9] consists of a set of peer-to-peer (P2P) Internet web services that support the creation of spontaneous multi-user collaborative sessions, allowing users to selforganise and communicate, share tasks, workloads, and content, and interact transparently using multiple different computing devices, networks, and operating systems. Coco is designed to handle both heterogeneity (interoperability between systems) and to scale from very small to very large communities using $\mathrm{P} 2 \mathrm{P}$ technologies.

Coco is built on a services-based architecture with collaborative community services hosted by JXTA peers and peer groups. Community data (such as profiles, portfolios, and participations) is represented using RDF data structures and exchanged by peers using the Coco metadata content service [10]. Synchronous interactions are supported by the Coco Messaging Service which allows groups of participants to engage in real-time video, audio, and IM interactions and the Interaction Service, which supports real-time presence notification within group interactions. The objective in providing distinct services is to allow collaborative community applications to access either all or a subset of Coco services hosted by participating peers.

To fulfill the need for enabling collaboration across a range of devices, we are developing a version of Coco aimed at mobile devices, such as PDAs and mobile phones. MicroCoco [11] is being developed using Java 2 Micro Edition (J2ME) [12] and JXTA for J2ME (JXME) [13] technologies. JXME interoperates with JXTA protocols allowing for mobile devices to participate in JXTA networks. The services provided by MicroCoco are a subset of the full platform. This is because the hardware constraints allow a limited amount of bandwidth for communication with other full Coco peers. For example, a group of Coco peers may be able to participate in video conferencing; however a MicroCoco peer running on a mobile phone may not necessarily have the bandwidth to deal with an incoming video stream, or the hardware capabilities to display that stream. A MicroCoco peer could however participate in sessions that require more asynchronous communications such as instant messaging, content sharing and co-authoring.

We assume that the mobile device will not be a user's primary computing device and have designed MicroCoco accordingly. To overcome some of the problems with mobile devices, we pair mobile peers with desktop peers. The mobile peer can then be used for particular tasks whilst away from the desktop, such as searching for content, whilst actually redirecting downloads to the paired desktop peer. This content redirection allows the user to still consume large amounts of content whilst using a constrained mobile device.

To support seamless collaboration across devices, we are implementing a session handoff mechanism that allows a user collaborating on one device to transfer session information to another device. The concept of Application Session Handoff (ASH) has been identified and practically demonstrated by UCLA's Pervasive Computing Laboratory project, iMASH [14]. In [14], the authors discuss the need for continuous 
computing - the ability to migrate application sessions between heterogeneous platforms. We plan on implementing this concept for collaborative sessions in Coco where some of the issues of dealing with platform heterogeneity are inherently solved through the use of Java and JXTA technologies.

\section{Conclusions}

This paper has presented a number of issues that reflect our experiences in the development of collaborative community systems. We submit that the goal of future community systems should be to support transparency via interoperability in the underlying architecture. Such transparency will enable a diverse range of community systems (including websites and applications) to access profile data, portfolios, and historic participations of participants and built community facilities by consuming collaborative services from a range of sources.

There are a number of potential areas of future work, including:

- Managing context switching - the implementation of a facility (possibly by via the incorporation of a new service into the design) that allows users to dynamically migrate from one device to another without affecting the continuity of collaboration.

- Support for user roles - Coco does not currently provide mechanisms for defining user roles within a collaborative context. Roles are, however, fundamental to effective collaborative and in future the platform needs to provide extended support for complex roles.

- Support for user activities - research into user activities, via case studies, will enable us to look more closely into the social aspects of online communities, particularly where mobile technologies are involved.

- Technological performances. Evaluating consistency of centralised vs. decentralised communities in terms of user-experience (speed of forming communities, development of roles, and socialisation).

\section{References}

1. Bardram, J. E.: Supporting Mobility and Collaboration in Ubiquitous Computing. Technical Report CfPC 2003-PB-38, Center for Pervasive Computing, Aarhus, Denmark, (2003).

2. Friendster, online community of friends: http://www.friendster.com, (2005)

3. Ryze, business networking: http://www.ryze.com/, (2005)

4. Marsic, I.: An Architecture for Heterogeneous Groupware Applications, Piscataway, NJ, USA: Rutgers University, (2000).

5. Mynatt, E. D., Adler, A., Ito, M., and O'Day, V. L.: Design for network communities. In Proceedings of the SIGCHI Conference on Human Factors in Computing Systems (Atlanta, Georgia, United States, March 22 - 27, 1997). S. Pemberton, Ed. CHI '97. ACM Press, New York, NY, 210-217.

6. Wiki, What Is Wiki: http://wiki.org/wiki.cgi?WhatIsWiki, (2005)

7. Microsoft Passport: http://www.microsoft.com/net/default.mspx , (2005) 
8. Bhana, I. Johnson, D. Alexandrov, N.: Supporting ad hoc Collaboration in Peer-to-Peer Networks", Proc. of 17 International Conference on Parallel and Distributed Computing, San Francisco (2004)

9. Bhana, I. Johnson, D.: A Peer-to-Peer Approach to Content Dissemination and Search in Collaborative Networks", Proc. of International Conference on Computational Science 2005, Atlanta (2005)

10. Bhana, I. Johnson, D.: Knowledge Management for Collaborative Learning, Proc. of the Interactive Computer-aided Learning 05 conference, Villach, Austria (2005)

11. Java 2 Platform Micro Edition (J2ME), http://java.sun.com/j2me/, (2005)

12. JXME: JXTA Platform Project, http://jxme.jxta.org/proxied.html, (2005)

13. Bagrodia, R., Bhattacharyya, S., Cheng, F., Gerding, S., Glazer, G., Guy, R., Ji, Z., Lin, J., Phan, T., Skow, E., Varshney, M., and Zorpas, G. 2003. iMASH: interactive mobile application session handoff. In Proceedings of the 1st international Conference on Mobile Systems, Applications and Services (San Francisco, California, May 05 - 08, 2003). MobiSys '03. ACM Press, New York, NY, 259-272. 